\title{
Study of the Relationship between Magnetic Helicity and Solar Coronal Activity
}

\author{
Kanya Kusano
}

Graduate School of Advanced Sciences of Matter, Hiroshima University

\begin{abstract}
Magnetic helicity, which is a measure of magnetic flux linking, is widely believed to play a crucial role for the solar coronal energetic processes, e.g. heating, flares and eruptions. In this paper, we introduce the several new findings of magnetic helicity physics in the solar corona both from the observational and theoretical points of view. The new observations based on the vector magnetograms successfully revealed that the solar coronal activity is indeed related not only to the intensity of magnetic helicity injection from the photosphere, but also to the complexity in the structure of magnetic shear. In particular, we recently found through the advanced analyses of vector magnetogram data that steep reversal of magnetic shear may efficiently activate the liberation of free energy stored in the coronal magnetic field. Motivated by the results, we developed the large scale three-dimensional simulation to investigate the causal relationship between the magnetic helicity injection and the energy liberation in the solar corona. The simulations clearly demonstrated that the reversal of the magnetic shear in a magnetic arcade can cause the sudden onset of plasmoid eruption. The mechanism is able to be explained as a self-exciting process of multiple magnetic reconnections. Finally, we propose a new scenario for the triggering mechanism of eruptive flares, which is called the reversed-shear flare model.
\end{abstract}

Keywords. Sun: coronal mass ejections (CMEs)

\section{References}

Kusano, K., et al., 2002, ApJ, 577, 501

Kusano, K., et al., 2004, ApJ, 610, 537

Maeshiro, T., et al., 2004, ApJ, submitted

\section{Discussion}

SUBRAMANIAN: What is the measure of structural complexity you use?

KusANO: Length of shear inversion layer where the sign of magnetic shear is changed.

LAKHINA: What is the magnetic Reynolds number used in your simulation?

Kusano: The magnetic Reynolds number is $10^{5}$ in our simulation except in the thin current layer, where it is reduced to $2 \times 10^{3}$ by anomalous resistivity. 